\title{
An ADS irradiation facility for fast and slow neutrons
}

\author{
Fabio Panza, ${ }^{* a}$, Gabriele Firpo ${ }^{b}$, Guglielmo Lomonaco ${ }^{c}$, Mikahail Osipenko $^{a}$, Giovanni Ricco, ${ }^{\text {,ad }}$, Marco \\ Ripani $^{\mathrm{a}, \mathrm{d}}$, Paolo Saracco ${ }^{\mathrm{a}}$ and Carlo Maria Viberti ${ }^{\mathrm{b}}$ \\ ${ }^{a}$ Istituto Nazionale di Fisica Nucleare - Sezione di Genova, V. Dodecaneso, 33 - 16146 Genova (Italy) \\ ${ }^{b}$ Ansaldo Nucleare C. so F.M. Perrone, 2516152 Genova (Italy) \\ ${ }^{c}$ DIME/TEC - University of Genova, V. all'Opera Pia, 15/A - 16145 Genova (Italy) \\ ${ }^{d}$ Centro Fermi - Compendio del Viminale - Piazza del Viminale 1- 00184 Rome (Italy)
}

\begin{abstract}
We report on the studies of an accelerator-driven subcritical nuclear research facility which can simultaneously provide a fast and a thermal neutron flux.

The conceptual design presented here starts from a $432 \mathrm{~kW}\left(\mathrm{k}_{\text {eff }}=0.96720 \pm 0.00052\right)$ ADS composed by 110 solid lead fuel assemblies each with size $9.7 \times 9.7 \times 87 \mathrm{~cm}^{3}$, filled with 81 MOX pins of 0.357 $\mathrm{cm}$ radius and surrounded by a $0.068 \mathrm{~cm}$ thick AISI steel cladding. Source neutrons are produced by a $(p, n)$ reaction from a $70 \mathrm{MeV} 1 \mathrm{~mA}$ proton beam impinging on a beryllium target [1].The core is helium cooled and is surrounded by a $80 \mathrm{~cm}$ lead reflector. Core and reflector are contained within a $2 \mathrm{~cm}$ steel vessel, as previously described in [2]. For $k_{\text {eff }}=0.97089 \pm 0.00067$ ) and $530 \mathrm{~kW}$ power, the system is composed by an assembly of 60 fuel elements identical to the original ones where:

- the Lead reflector has been replaced by three concentric ring layers, the first made of $25 \mathrm{~cm}$ lead followed by $50 \mathrm{~cm}$ graphite and $10 \mathrm{~cm}$ lead.

- in the cooling system helium has been replaced by light water running in larger pipes $(0.5 \mathrm{~cm}$ diameter)

We simulated the neutron flux in three core positions (internal, medium, external) and in two graphite reflector positions (internal, external), finding that the flux is still mostly fast in the core, while it exhibits a strong thermal component in the reflector.

Two extraction channels have been designed one of thermal neutrons from graphite and the other of direct source neutrons
\end{abstract}

[1] M.Osipenko et al, Measurements of neutron yield... Nucl. Instr. Meth A723 (2013)

[2] Focus Point, An intrinsically safe facility edited by G.Ricco Eur. Phys. J. Plus 129 (214) 